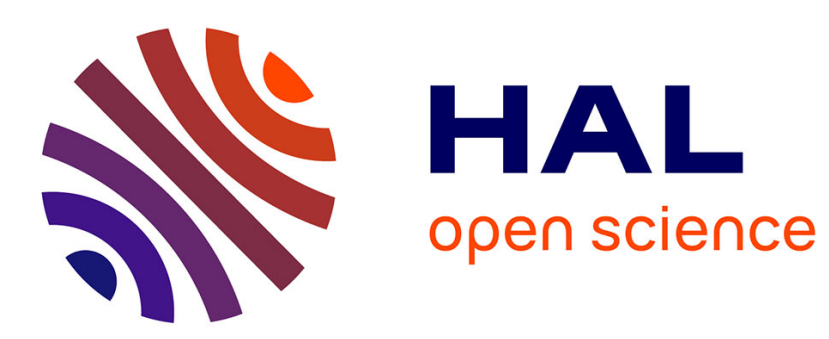

\title{
FROM THE ATOMIC STRUCTURE OF INTERFACES TO THEIR PHYSICAL AND CHEMICAL PROPERTIES
}

\author{
A. Bourret
}

\section{> To cite this version:}

A. Bourret. FROM THE ATOMIC STRUCTURE OF INTERFACES TO THEIR PHYSICAL AND CHEMICAL PROPERTIES. Journal de Physique Colloques, 1990, 51 (C1), pp.C1-1-C1-10. 10.1051/jphyscol:1990101 。 jpa-00229996

\section{HAL Id: jpa-00229996 https://hal.science/jpa-00229996}

Submitted on 1 Jan 1990

HAL is a multi-disciplinary open access archive for the deposit and dissemination of scientific research documents, whether they are published or not. The documents may come from teaching and research institutions in France or abroad, or from public or private research centers.
L'archive ouverte pluridisciplinaire HAL, est destinée au dépôt et à la diffusion de documents scientifiques de niveau recherche, publiés ou non, émanant des établissements d'enseignement et de recherche français ou étrangers, des laboratoires publics ou privés. 
FROM THE ATOMIC STRUCTURE OF INTERFACES TO THEIR PHYSICAL AND CHEMICAL PROPERTIES

\author{
A. BOURRET \\ Département de Recherche Fondamentale, Service de Physique, Groupe \\ Structure, CEN-Grenoble, F-38041 Grenoble Cedex, France
}

\begin{abstract}
Résumé - De nouveaux résultats expérimentaux ont été obtenus récemment sur la structure atomique des joints de grains et des interfaces. Les différentes techniques donnant des renseignements à 1 'échelle atomique sont exposées : 1a microscopie électronique à haute résolution, la microscopie à émission de champ, la microscopie tunnel et la diffraction de rayons- $X$. La comparaison de ces résultats avec les modélisations sur ordinateur et le lien avec les propriétés des joints ont été menés à bien dans quelques cas particuliers. C'est un des enjeux majeurs des prochaines années que de généraliser ces comparaisons dans l'étude des interfaces.
\end{abstract}

Abstract - In the recent past years experimental new results have been obtained on the atomic structure of grain boundaries and interfaces. The different techniques for recording atomic-scale information at boundaries, either topological or chemical, are critically reviewed. These include high resolution electron microscopy, atom-probe field-ion microscopy, scanning tunneling microscopy and X-ray diffraction. The confrontation with computer modelling as well as the link with the boundary properties has been carried out in few cases. It is emphasized that such a comparison is one of the major issue of the interface studies in the next future.

\title{
1 - INTRODUCTION
}

The study of grain boundaries and recently of more general interfaces has been the subject of many experimental as well as theoretical works. For some recent reviews see for instance $/ 1,2 /$. A continuous progress of our understanding of intergranular and interphase boundaries properties is evident when one follows the litterature on the subject. However the recent years seem to be characterized by the development and the proper use of new techniques at atomic scale. Among others the most advanced are i) the high resolution electron microscopy (HREM) $/ 3 /$, ii) the field ion microscopy with the associated atom-probe $/ 4 /$, $i j i$ )the very recent applications of tunneling microscopy to interfaces $/ 5 /$. Thanks to these various techniques the experimentalist has now in principle direct access to atomic positions in the lattice, to the atom chemical species and/or to the electronic structure via the electron density of states. In addition $X$-ray diffraction techniques could give the atomic positions at periodic interfaces with a small unit cell. This recent trend towards atomic-scale probing is likely to be even more developed in the near future. The reason is that simultaneously the last few years have seen an intensive use of the computer simulation. More sophisticated interaction potentials as well as larger computer capabilities have boosted large amount of information on the structures and the properties of various boundaries. These informations should now be confronted to the experience, hence demanding more experimental results from the techniques at atomic scale. As this confrontation is likely to be one of the major issue of the next decade it seems important to review the main recent experimental results obtained with the atomic-scale technique and their impact in the understanding of the main physical properties of intergránular and interphase boundaries.

\section{2 - PROGRESS IN THE ATOMIC SCALE TECHNIQUES}

The advantages and limitations of the different methods are critically described with specific examples on different kinds of boundaries. Some of their characteristics are summarized on Table 1. 


\begin{tabular}{|c|c|c|c|c|c|}
\hline Technique & $\begin{array}{l}\text { Resolution } \\
\text { (structura? } \\
\text { information) }\end{array}$ & $\begin{array}{l}\text { Resolution } \\
\text { (chemical } \\
\text { information) }\end{array}$ & $\begin{array}{l}\text { Probed } \\
\text { thickness }\end{array}$ & $\begin{array}{c}\text { Material } \\
\text { limitations }\end{array}$ & $\begin{array}{c}\text { Specimen } \\
\text { Requirements }\end{array}$ \\
\hline$H R E M$ & 0.16 to $0.2 \mathrm{~nm}$ & $\begin{array}{c}\text { directly: none } \\
\text { indirectly: } 1-2 \mathrm{~nm} \text {, } \\
0.4 \mathrm{~nm} \text { in very } \\
\text { favorable case }\end{array}$ & $\begin{array}{c}\text { Integrated } \\
\text { over } 10-20 \mathrm{~nm}\end{array}$ & $\begin{array}{l}\text { Radiation } \\
\text { damage } \\
\text { in ionic } \\
\text { materials }\end{array}$ & $\begin{array}{l}20 \text { nm thin } \\
\text { foil with } \\
\text { boundary } \\
\text { end-on }\end{array}$ \\
\hline $\begin{array}{c}\text { Atom-probe } \\
\text { F I M }\end{array}$ & 0.3 to $0.4 \mathrm{~nm}$ & $\begin{array}{l}0.4 \mathrm{~nm} \text { (atomic } \\
\text { sensitivity) }\end{array}$ & $\begin{array}{c}\text { Surface method } \\
\text { (peeling layer } \\
\text { by layer } \\
\text { possible) }\end{array}$ & $\begin{array}{c}\text { Best in } \\
\text { non-ductile } \\
\text { materials }\end{array}$ & $\begin{array}{c}\text { Wire with tip } \\
\text { radius } \\
10-100 \mathrm{~nm} \\
\text { High density } \\
\text { of boundaries }\end{array}$ \\
\hline$S T M$ & $\begin{array}{l}0.2 \mathrm{~nm} \text { Tateral } \\
0.005 \mathrm{~nm} \text { normal }\end{array}$ & $0.2-0.3 \mathrm{~nm}$ & Surface method & $\begin{array}{l}\text { Insulators } \\
\text { are inappro- } \\
\text { priate }\end{array}$ & $\begin{array}{l}\text { Flat and clean } \\
\text { surface with } \\
\text { boundary end-on }\end{array}$ \\
\hline $\begin{array}{c}\text { X-ray } \\
\text { diffraction }\end{array}$ & $\begin{array}{l}0.002 \mathrm{~nm} \text { later } \\
0.006 \mathrm{~nm} \text { norma }\end{array}$ & $\begin{array}{c}\text { indirectly } \\
\text { possible }\end{array}$ & $\geq 5 \mathrm{~nm}$ & none & $\begin{array}{c}\text { boundary } \\
\text { running parallel } \\
\text { to the surface } \\
\text { at few } 10 \mathrm{nms}\end{array}$ \\
\hline
\end{tabular}

Table 1 - Main characteristics of the atomic-scale sensitive techniques : high resolution electron microscopy (HREM), atom-probe field-ion microscopy (FIM), scanning tunneling microscopy (STM) and X-ray diffraction.

\subsection{High resolution electron microscopy}

The latest generation of intermediate-voltage high resolution electron microscopes gives the exciting prospect of resolution limits below $0.2 \mathrm{~nm}$. Moreover under specific well-defined conditions it is even possible to deduce useful structural details down to small fractions of the resolution limit.

The principle of any interface observations is to observe at least one but preferably both crystals at each side of the boundary, along a zone-axis with the boundary seen end-on. This geometry assures that atomic columns will be well aligned on either side of the boundary giving an interpretable 2-D projection of a 3-D crystal. The information is always integrated along the observation axis, $z$, over the specimen thickness. The interpretation even in this case is rarely direct and a comparison with computer simulated images is usually necessary. At the boundary itself several cases should be considered as the 2-D medium is projected to a 1-D image :

i) all displacements are confined in the plane perpendicular to the observation axis and does not depend on the $z$ coordinate : the relaxation at the boundary could be uniquely determined by one observation axis $/ 6 /$. It is also necessary in order to get atomic-scale information, that the distances between atomic columns are larger than the resolution limit. This is the case, for instance, for pure tilt grain boundaries (GBs) along low index zone-axis.

ii) If the displacements along the $z$-direction are non zero but remain independant of $z$, several HREM images taken along different observation axes could be taken $/ 7 /$.

iji) When the displacements are more general and varie in any direction (case of a twist grain boundary) atomic-scale information is much more difficult to obtain. A series of projections along different observation axes combined with a 3-D reconstruction scheme would be necessary. It is in principle possible but it would require a resolution 1 imit smaller than the one presently available. However HREM images, in this case, give already interesting information about the periodicity at the interface and the presence of additional defects (steps, ledges, facets and dislocations...) $/ 8 /$.

In the most favorable cases a complete crystallographic analysis has been performed including the 2-0 space group determination as well as the atomic coordinates of several atoms in the basic unit cell of the boundary itself. The accuracy of the rigid-body translation determination is of the order of $0.01 \mathrm{~nm}$ and the atomic coordinates are measured within \pm $0.02 \mathrm{~nm}$ (about one tenth of the resolution limit) $/ 9 /$. All these studies are static structure determinations. Two points should deserve more attention in the future: i) the influence of the atomic vibrations at the boundary and $i j)$ the eventual observation of dynamic features directly in-situ. Concerning the first point, up to now the atomic vibrations were neglected in the image interpretation apart from the usual Debye-Waller factor attenuation in the atomic scattering. However in a boundary some bonds could be weakened enough so as to affect the amplitude of atomic vibrations even close to the room temperature. Concerning the second 
point, it is now feasible to use a hot stage at high temperature with a good resolution /11/. Results are still quite scarce but should appear in the next feature.

In addition to structural information, some chemical information is al so available from HREM images. That type of analysis is becoming quite popular although it relies on relatively indirect information : mainly the atomic radius differences and/or some Z-contrast due to the difference between atomic scattering factors. The method which is employed, assumes a large amount of a-priori information : i) the atomic species which are involved are already known but their exact location is to be found; $i$ i) the relationship between the lattice constant and the composition is supposed to be known or has been measured independantly; iij) the image pattern should be uniquely and if possible linearly dependant on the chemical composition. Although limited, this technique has been successfully employed in multilayer structures $/ 12,13,14,15 /$.

The main limitation of the HREM technique relies on the projection problem. The information is always averaged (in a non-linear manner) over the specimen thickness. As a consequence there is a reduction by one dimension in the available information which is not simply overcome. The end-on geometry is the most popular way of overcoming it, but it should be associated with at least two or more projection axes. Several trials of planar geometry, in which the boundary is observed perpendicular to its habit plane, are not completely convincing. The main difficulty here is due to the large contribution to the image of the upper and lower parts. Two other limitations should deserve some attention al though less serious: the relaxation at both surfaces of the thin layer obtained after specimen preparation and the radiation damage. The relaxation is only serious in strained boundaries such as those produced in multilayers. On the other hand the radiation damage could be serious : low- $Z$ specimens or crystal with an important ionic bonding are generally more sensitive. However each specimen is a specific case and a general assessment is difficult to put forward.

In summary the main applications of the HREM technique concern $i$ ) the periodicities at the interface, $i i)$ the coherency or the loss of coherency with or without interfacial dislocations, $i j i)$ the atomic modeling and positionning at the interface, iv) the roughness of the boundary and the presence of steps, facets or chemical gradients.

\subsection{Field ion microscopy (FIM) and atom-probe FIM}

With the FIM it is possible to form an image of the arrangement of individual atoms on the surface of a small rounded tip and in conjunction with a time-of-flight mass spectrometer to identify them chemically /4/. However it should be realized that the atomic imaging is restricted to kink or ledge sites at the boundary as well as in the two crystals on either side of the boundary. Controlled field evaporation, atomic layer by atomic layer, extends this inherent surface technique to a bulk technique : it enables to explore an interface along the boundary plane. The lateral resolution is theoretically determined by the tip temperature and the tip radius which should be as low as possible. It is of the order of $0.3 \mathrm{~nm}$ in the optimum case (tungsten) and therefore does not allow to make a crystallographic analysis of the boundary structure. The resolution normal to the surface is substantially better and of the order of $0.03 \mathrm{~nm}$. However any quantitative measurement is hintered by unknown surface relaxation under the applied electric field. As a consequence although the detection of GBs geometry in very small grain size specimens is easy to perform, the boundary structure has never been determined by FIM. Nevertheless interesting parameters such as the misorientation, the GB plane and the presence of additional defects could be deduced from an FIM picture. This method could be applied to a general grain boundary (twist as well as tilt) in a random orientation and in materials containing a very high defect density $/ 17 /$. The same is true for an interphase interface although its range of applicability is restricted to the few cases where both phases exhibit a ring structure at the same applied voltage. These limitations combined with the fact that GB-migration could be induced in non-refractory metals and that field evaporation occurs preferentially at boundaries, has impeded extensive use of the FIM for structural investigation of boundaries in the last decade.

Fortunately the facility for identifying the chemical nature of atoms at near atomic level has renewed the interest for the atom-probe FIM. This consist of the chemical identification of a single ion emitted from a selected area of the tip by small field evaporation pulse. The counting being atom per atom the statistical significance of atom-probe data has to be improved by data accumulation which is sometimes cumbersome but yields to unique data on impurity segregation at grain boundaries $/ 10,16,19 /$ interfaces $/ 20 /$ or stacking faults $121,22 /$. 
Despite its potential interest, specially with the simultaneous use of FIM imaging and atomprobe chemical capabilities, these techniques have not attracted very much attention and it ought to be more widely exploited.

\subsection{Scanning Tunneling Microscopy (STM)}

The STM is an high-resolution instrument capable of structural information as well as electronic and spectroscopic imaging. This provides a unique versatile tool but also sometimes its rather subtle image interpretation. The tunneling current probes the electronic density of states (occupied or unoccupied depending of the applied voltage) of the substrate convoluted by those of the tip. If the tip wave function is supposed to be known, the tunnel current, I, could be related to the topology (position in the space) as well as to the electronic state of one particular atom at the surface. The resolution is $0.2 \mathrm{~nm} 7 \mathrm{aterally}$ and $0.005 \mathrm{~nm}$ normal to the surface. Topographic images are generally taken with scans at constant tunneling current monitoring the change in the distance between tip and substrate and electronic or spectroscopic images are performed at steady tip on a particular atom by measuring the $I(V)$ characteristic $/ 5 /$. The combination of all these signals by a clevel electronically-driven biasing may provide direct and selective imaging of atomic species particularly on semiconductor materials where tunable-laser excitation is al so possible $/ 23 /$.

The observation of an interface is limited to the edge-on geometry, only the surface atoms being probed. Very few applications are yet published the technique being still in its infancy. However one could envisage to look at a variety of intergranular and interphase boundaries with the exception of insulating materials. The chemical information through some recognizable feature in the density of states is rather indirect when compared with the atom-probe FIM, but there is a unique capability of combination between the structural information and the electronic information. This should attract many new developments in employing this technique to study specific segregation sites in a boundary at an atomic scale, or to recognize the location of different species at a boundary specially in alloys.

Unfortunately the precise determination of the atomic coordinates at the interface is difficult owing to possible surface relaxation as well as to spatial distorsion due to the drift. Recently this instrument has been extended to probe a buried interface with $1 \mathrm{~nm}$ spatial resolution through ballistic electron spectroscopy measurement $/ 24 /$. This applies to metalsemiconductor interfaces.

\subsection{X-ray diffraction}

Quantitative $X$-ray diffraction techniques could be used directly or through standing-wave, to study interfaces. Strictly periodic interfaces such as those produced in a commensurate grain boundary could give rise to measurable reflections in reciprocal space : the interface reflections are found to be modulated rods of intensity extended perpendicular to the interface $/ 25 \%$. The availability of high flux X-ray sources at synchrotron facilities has enable to measure the intensity of the GBs reflections. The atomic structure can then be obtained by testing model structures with a $x^{2}$ analysis. The number of required reflections increases with the number of atoms included in the unit cell determined by the coincidence site lattice (50 were measured for 8 independant atomic positions in $\Sigma=13 \mathrm{~GB} / 26 \%$. The resolution is very good being $\pm 0.006 \mathrm{~nm}$ normal to the interface and $0.002 \mathrm{~nm}$ parallel to the interface. Care should be taken in order to el iminate contributions from double diffraction as well as ordinary forbidden reflections appearing in thin layers. Integrated intensities of the $1=0$ $p l$ ane give the projected location onto the $G B$ plane while the relrod intensity profile along each specific rod gives an information in the position perpendicular to the GB plane. Such experiments were limited up to now to the transmission case in which the interface plane runs parallel to the surfaces of a thin specimen. That type of geometry is therefore 1 imited to hot pressed boundaries. A great advantage of this technique is the availability to solve any periodic structure in particular twist grain boundaries which are more difficult to study by HREM. However it should be generalyzed to less specific sample geometry. For instance a grazing incidence in the reflection mode could broaden the applications to near surface interfaces such as those produced after surface reaction or deposition. Another method is the $X$-ray standing waves which could give structural information with a $z$-resolution 1 or $2 \%$ of the d-spacing. This method may be used to locate at interfaces specific atoms recognized by fluorescence yield measurement $/ 27,28 /$. This method can be applied to multilayer in order to locate a specific species and make extended X-ray absorption fine structure measurement $/ 29 /$ : in such a case the resolution is limited to the dimension normal to the multilayer interfaces and is of the order of $10 \%$ of the multilayer period. 


\section{3 - POSSIBLE COMPARISON WITH COMPUTER MODELLING OF STATIC STRUCTURE}

The comparison between experimental results at atomic level and the computer modelling has been made on few examples. This comparison has itself several degrees of complexity : one may compare the rigid body translations at grain boundaries as a first overall parameter ; then one may compare the most stable structures when multiple structures are possible and finally one may compare the atom coordinates. The comparison has been so far limited to the first two points with the HREM technique and includes all these points with the $X$-ray technique. The first example considered is the $\Sigma=3$ (211) twin boundary in Ge (and Si), a tetracoordinated structure. This $G B$ was shown $/ 30 /$ to be reconstructed forming a $\mathrm{cm}(2 \times 2) \mathrm{struc}$ ture and having an important rigid-body translation (RBT). Several types of interatomic potential were tried. The simplest is the modified Keating potential used by Mauger et al 131/ which already agrees with the measured RBT in the direction parallel to the GB plane, but has a smaller expansion (by a factor 2) than the measured one. However the accuracy in this experimental determination is not good enough to decide if this discrepancy is meaning ful1 or not. A more stringent test is given by identifying the lowest energy configuration among the five different reconstruction scheme. In silicon the use of semi-empirical tight binding $/ 32 /$ as well as Keating-type /33/ potential has shown that indeed the $\mathrm{cm}$ (2x2) structure was the lowest in energy. It is worthwhile to note that the energy difference is extremely small and this agreement between experimental and calculated results is a remarkable achievement.

As a further example recent results on $<001\rangle$ pure tilt GB in germanium were compared with computer relaxed configurations using two types of empirical interatomic potential : the Keating-type potential and the Tersoff potential /34/ (Fig. 1). For a large number of GBs the agreement on the final configuration (state of the lowest energy) is good : this is the case for $(310) \Sigma=5,(320) \Sigma=13$ and remarkably for $(11,3,0) \Sigma=65$ which has a 1 arge variety of possible unit mixing. Differences as small as a few per cent in energies are calculated and at least hierarchy in energy agrees with the observation. However in the case of $(510) \Sigma=13$ multiple structures are observed : one of the observed configuration is not directly predicted from computer simulated static calculation. The same is true for (710) $\sum=25$ and (910) $\Sigma=41$ GBs for which the observed structure presents an extremely variable core with configurations having relatively high static energy. It has been proposed /35/ that the configurational entropy term could explain this discrepancy.
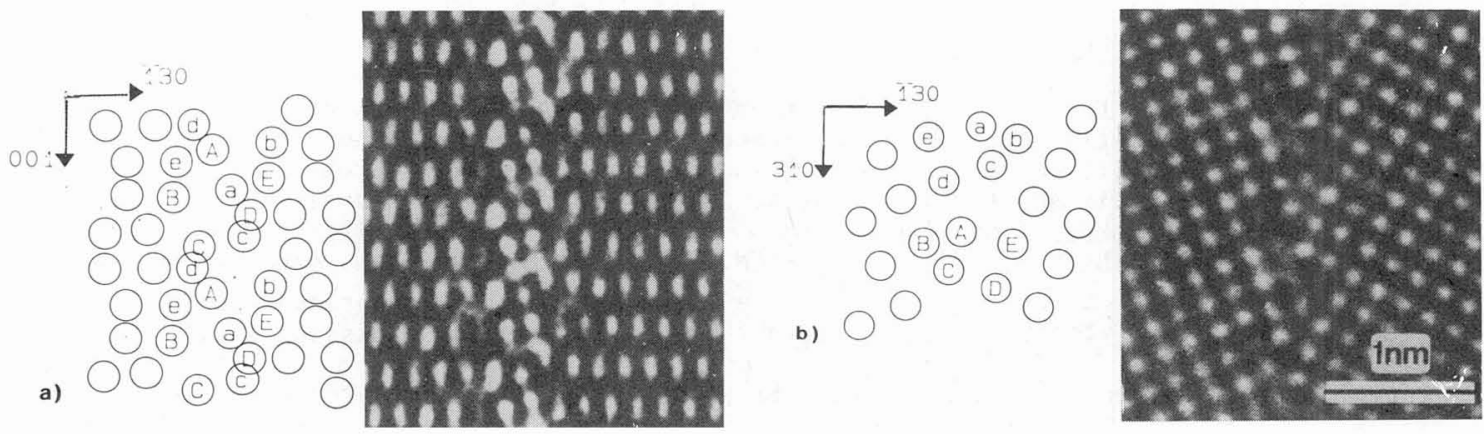

Fig.1. - Experimental images of a (130) $\Sigma=5$ tilt GB in Ge as compared with the computer relaxed structures along two projection axes. From $/ 7 /$.

In metals although a large variety of computer simulated structures are available since twenty years oniy few comparison with experimental results were made. For the case of (331) $\Sigma=19$ tilt in gold, Krakow et al /36/ showed that a close agreement between observed and computer relaxed structures exists. The RBT and the large expansion agrees well with the calculated ones. Unfortunately two very dissimilar structures have the same [110] projection and could not be distinguished by HREM. In molybdenum the $(810) \quad \Sigma=65$ HREM images were compared with relaxed structures using Mie-type potential /37/. The calculated energies for two variants of a (013) structural unit (empty or non empty core) are extemely close to each other and multiplicity is predicted $/ 38 /$. This is confirmed experimentaliy although the empty form with a capped triangular prism is the most frequently encountered. However the analysis is made questionable because of the possible oxygen segregation at GB which is likely in molybdenum. A further example of a qualitative comparison between experiment and computer relaxed structures is given in NiO by Merkle and Smith /39/. Large amount of open space was observed as predicted in symmetric section of (210) $\Sigma=5$, similariy the (320) $\Sigma=13$ aqrees quite well with the calculated structure. However asymmetric facets with low index 
grain boundary plane are present in the same (320) $\Sigma=13$. These structures are more compact than the symmetric ones and may have lower energies. This is clearly an example where new configurations, which were not previously thought of, have been suggested by the experimental observations and should be included in computer relaxation studies.

The most precise comparison is undoubtedly the one performed on $\Sigma=5$ [001] twist GB in gold, as it applies directly to the atomic coordinates. It has evidenced distorted octahedra and tetrahedra associated with atomic rumpling of the atoms at different distances from the GB plane /25/. This. structure was not predicted by computer simulation al though some overall parameters like the total expansion and the rumpling are qualitatively in agreement. More recently the same analysis was performed on $\Sigma=13$ [001] twist GB in gold /26/. It confirms the earlier hypothesis that computer relaxation is unable to predict the exact atomic location in the first and even the second atomic plane from the GB plane. Absolute differences have an average value of $0.024 \mathrm{~nm}$ and could be as large as $0.042 \mathrm{~nm}$. One possible explanation for this large discrepancy is the unreliability of empirical pair potential in regions where the density falls down by more than $8 \%$. Some authors have suggested /40/ that a mixture of different structures (multiplicity model) could explain the X-ray data. However the reliability factor is not improved by such a mixture. Recently Balluffi et al. have questioned the $\Sigma=5$ [001] large displacements $/ 18 /$ and found results much closer to the computer relaxed structure. Therefore it is urgently necessary to confirm these results on different materials and see if this behaviour can be generalized.

\section{4 - ATOMIC SCALE INFORMATION AND BOUNDARIES PROPERTIES}

The understanding of the correlation between the atomic structure and physical properties of boundaries has been one of the major goal of the past studies. However although the dislocation approach has been very successful in the physical understanding of low angle grain boundaries, there is not yet unifying concepts which could be applied to more general interfaces. Some new ideas are emerging in different fields but they are still rather 1 imited due to the formidable task which consists of making a link between one specific defect of the crystal at atomic scale with an overall property depending on many body interactions. Various fields wi11 be rapidly reviewed : thermodynamical properties, impurity segregation electronic properties and some mechanical properties at specific boundaries for which a 1 ink with atomic structures has been at least tempted to be established.

\subsection{Thermodynamical properties}

Although interest in the thermodynamical properties of boundaries has received wide spread attention from theoreticians, only few experimental results were obtained at an atomic scale. The phase transition between faceted and a rough asymmetric GB was directly observed but at a scale which is not the atomic scale /41/. Moving grain boundaries in gold as well as nucleation or decomposition of existing ones have been recorded /11/ showing that phase transition could be observed in-situ by HREM or STM. Similarly the evidence obtained by Leroux et al /42/ of a wetting of antiphase boundaries by the disordered phase in CoPt 3 although not yet at an atomic scale is relevant to the techniques discussed here.

Multiple structures were evidenced in $\mathrm{Ge} / 35 /$ at an atomic scale and a core structure which contains several stable positions with many variants can be built. Therefore it has been emphasized that the total static energy can be misleading if the entropy term is not included. This could explain why the static energy configuration seems to be not the right parameter. These atomic scale observations suggest that phase transitions with order-disorder transformation could occur, although they were not yet observed.

The measurement of the boundary X-ray reflections as a function of temperature /43/ has been used to evaluate the thermal expansion coefficients of the $\Sigma=13$ [001] twist GB in gold, and the mean square displacement. The thermal expansion coefficient parallel to the GB is equal to the bulk value, while it is three times larger perpendicular to the GB. The mean square displacement is found to be $40 \%$ larger in the GB than in the bulk at room temperature. Although this experimental result is very important it remains now to make the connection of this behaviour with the form of the interatomic potential, a work which has recently been tackled by Sutton $/ 57 /$.

As a matter of fact the results are still very scarce and not fully exploited : however although often qualitative, the experimental results point to an important direction. The atom at the GBs have different properties when compared to the bulk: the mean square displacement is higher and probably very anisotropic, and the entropy term is modified by new configurational as well as vibrational terms. 


\subsection{Impurity segregation and chemical gradient}

The most clear atomic scale studies of interface segregation has been obtained by the atom probe FIM : on a stacking fault in $\mathrm{Co}-\mathrm{Nb}$ and $\mathrm{Co}_{0}-\mathrm{Fe}$ alloys /21/ or at GBs in austenitic stainless steel /10/ and in Mo-Re alloy /16/. Solute atom segregation of $\mathrm{Nb}$ or $\mathrm{Fe}$ is surprisingly high and limited to the SF plane. A tendancy to form $\mathrm{CO}_{3} \mathrm{Fe}$ plane composition is evidenced associated with an order-disorder transformation within the plane of the fault. This work demonstrates very conclusively that the elastic strain energy does not play as major role in the driving force and that interactions up to the third near neighbor atoms should be taken into account. In stainless steel boron was demonstrated to segregate at general GBs but not at coherent twins. The binding energy of boron to the GB was estimated at $0.65 \pm 0.04 \mathrm{eV}$. Experimental results were so rich that a detailed diffusion model has been proposed $/ 10 /$.

In molybdenum the HREM observations on pure GB have motivated the search for specific sites for oxygen segregation $/ 38 /$. It was predicted that several sites were possible : interstitially in the open core or substitutionnally with different interaction enthalpy. These predictions could have important implications in the theoretical modelling of interface segregation, although they have not yet been confirmed experimentally.

There is a number of HREM observations of interphase boundaries. See for instance the systems such as : $\mathrm{Al} / \mathrm{Ge} / 44 /, \mathrm{Cu} / \mathrm{Au} / 45 /, \mathrm{A} 1-\mathrm{Cu}-\mathrm{Li} / 13 /$, superalloys $/ 46 /, \mathrm{Mg} 0 / \mathrm{Al} / 47 /, \mathrm{Nb} /$ $\mathrm{Al}_{2} \mathrm{O}_{3} / 48 /, \mathrm{Cu} / \mathrm{Al}_{2} \mathrm{O}_{3} / 49 /$, and all the silicon-silicide interfaces, multilayers, quantum wells (for a review see $/ 3 /$ and $/ 50 /$ ). Chemical gradients close to the interfaces were also directly evidenced in few cases. Kazmerski /23/ in highly doped (B on Al) polycristalline silicon was able to show directly by STM that $B$ and $A$ l atoms were attracted close to the GB in a region $\sim 10 \mathrm{~nm}$ wide with no special segregation at the GB plane (here an asymmetrical $\langle 110\rangle 28^{\circ} \mathrm{tilt}$ ). As expected $\mathrm{B}$ and $\mathrm{Al}$ are substitutional. The addition of hydrogen (also made visible by spectroscopic means) introduces $\mathrm{Si}-\mathrm{H}$ bonds and $\mathrm{Al}-\mathrm{H}$ bonds but no direct interaction with $B$ atoms. A similar study in CuInSe, polycristal shows a very clear oxygen segregation at the grain boundary, the oxygen binding being preferentially at the indium sites. In multilayers structure which are frequently highly thermodynamical unstable systems, chemical gradients at interfaces could be produced either during the crystal growth or during post-annealing. In the $\mathrm{HgCdTe} / \mathrm{CdTe}$ multilayer system Kim et al were able to study the low temperature interdiffusion of $\mathrm{Hg}$ at an atomic scale /5l/. These authors used the digital pattern recognition to chemically map the mercury content on HREM images/12/ and demonstrate that the stability of a specific layer may depend sensitively on its depth from the surface, with a higher diffusivity close to the surface. In addition non-linear diffusion was necessary to introduce in order to explain the profiles. In the Fe/Ti system a chemical gradient at the interface was also evidenced by d-spacing measurement at a $1 \mathrm{~nm}$ scale /52/ (Fig. 2). A marked asymmetry between the two sides of a given layer is measured, which is explained by the growth process inherent to ion-sputtered deposited layers.
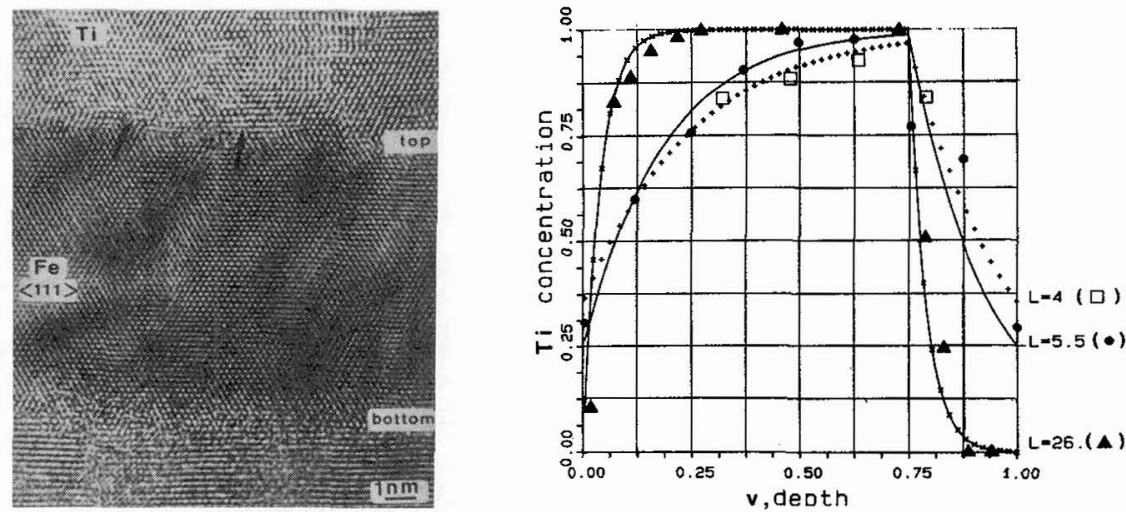

Fig.2 - Atomic image of a Fe/Ti multilayer with a $47 \mathrm{~nm}$ period, and the chemical profiles at different reduced period ( $L=26$ corresponds to $47 \mathrm{~nm}$ ).

Finally such chemical gradients are also evidenced at precipitates /53/. Fujita et al have shown that $\theta$, phase in Al-Cu dilute alloys is surrounded by a buffer layer. In addition the composition tends to vary slowly when crossing the interface. It is suggested that the intermediate layers are reminiscent of the GP-2 zones composition. Therefore contrary to the usually invoked denuded zone, an enriched layer could be stabilized in order to relax some of the elastic strain in a precipitation process. 
The above examples show how important will be the atomic scale observations with chemical mapping in order to understand and modelized correctly the segregation and the precipitation as well as the diffusion processes in highly metastable conditions.

\subsection{Electronic properties and atomic structures}

From the atomic structures deduced by HREM several electronic structures were calculated in semiconductors $/ 31,32,33 /$. They all agree on the following points (Fig. 3) :

- The density of states at grain boundaries does not contain deep levels inside the band gap in fully reconstructed structures.

- No localised states above the minimum of the valence-band and below the maximum of the valence band are calculated.
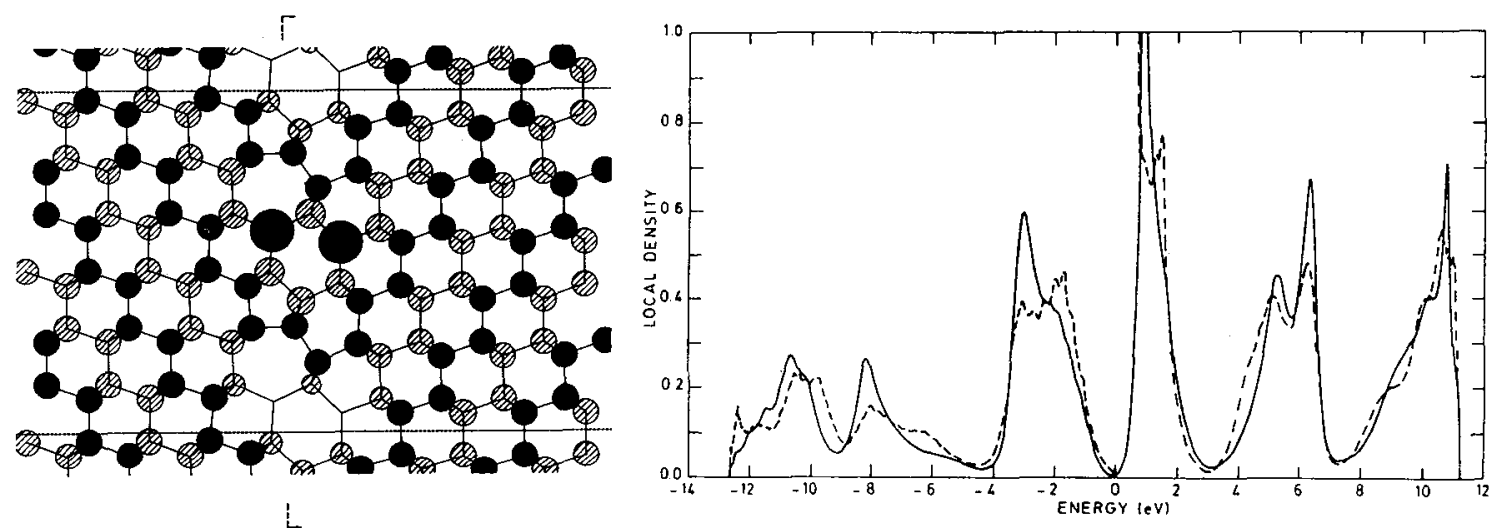

L

Fig. 3 - Atomic model of a (211) $\Sigma=3$ tilt GB in Ge deduced from HREM observations and the calculated total density of states in the perfect lattice (solid curve) and in the $G B$ (dashed curve). From $/ 31 \%$.

These results however appear to be in disagreement with experimental measurements of the tail states /58/ and the most plausible explanation is that most of the GB electrical activity is due to impurity segregation or possibly to additional point or line defects. Such an impurity effect was clearly demonstrated on copper segregated GBs in silicon by DLTS measurement /54/. In semiconductor polycristalline oxides (Mn-Fe ferrites) the correlation between the $G B$ structure and the in-situ voltage drop-off has been measured /55/. In particular the presence of a $1 \mathrm{~nm}$ glassy film double the voltage drop-off when crossing the barrier but most remarkably the general boundary are much more resistive than particular twin orientations.

As a consequence the correlation between the atomic structure of boundaries and the electronic or electric properties, is far from being understood. The sensitivity of the electrical measurement is so high that a direct correlation with atomic models at a scale close to a point defect is, for the moment, unrealistic.

\subsection{Mechanical properties}

The hardening role of the $G B$ and the mechanism by which dislocations could be incorporated at $a G B$ has been investigated recently by Elkajbaji and Thibault-Desseaux $/ 56 /$. It is shown that intrinsic dislocatiuns enter the $G B$ where they decompose into discrete $G B$ dislocations : these residues can migrate and react along the interface giving rise to macroscopic rotation of the adjacent grains and new residues. These mechanisms are observed at atomic scale in silicon by HREM. Although not made in-situ but by a frozen-in method, they provide a good basis for describing the absorption and the reemission of dislocations by GBs and could be in the future correlated with bulk mechanical properties.

5 - CONCLUSION

The development in parallel of experiments at atomic scale and of computer simulation is certainly one of the major issue of the next years. The first examples of such a comparison which have been carried out so far, have always shown the mutual benefit it can bring.

On one hand the experimental results have given surprising results which were not envisaged before. One may mention several examples : 
- The bond reconstruction with supercell formation at periodic interfaces.

- The presence of disordered structures in a "periodic" structure.

- The segregation at stacking faults in dilute alloys.

- The peculiar diffusion properties of multilayers.

It is, of course, necessary to confirm and extend these observations. The atomic scale techniques are generally highly sophisticated and the interpretation is often rather elaborate. The degree of confidence is sometimes relying on a sophisticated interpretation and progress should be made particularly in the case of STM in the understanding of the atomic images. Another severe limitations of all these atomic-scale techniques is their lack of ability to be applied to a large variety of material or to general boundaries. This generally prevent from mutual control by two or more different techniques.

On the other hand computer relaxations have given many new ideas which were confirmed latter by atomic-scale experiments. This was the case for :

- The presence of multiple structures.

- The prediction of several low energy interfaces.

- The existence of phase transition.

- The presence of several specific sites for segregation at grain boundaries.

The development in the computers capacity and speed will certainly tend to improve the quality of the interatomic potentials. A check, even on ab-initio calculations, will be however always necessary and atomic-scale experimental methods will be highly desirable.

As a conclusion it should be added that while it can be foreseen that this mutual confrontation is the future major development on studies of all kind of interfaces, it will be necessary to develop unified models and concepts. They have to give simple and insightful description of the physical properties of boundaries from microscopic mechanisms.

\section{REFERENCES}

/1/ Journal de Physique, Colloque C5, supp1. $n^{\circ} 10 \underline{49}$ (1988).

12/ Polycristalline Semiconductors, Grain Boundaries and Interfaces, Edit. by Möller, H.J. Strunk, H.P. and Werner, J.H., Springer Proc. in Physics 35, Springer-Verlag Berlin (1989)

13/ Bourret, A., in Characterization of the Structure and Chemistry of Defects in Materials, Ed. Larson B.C. Rühle M. and Seidman D.N., Vol. 138, Material Research Society, (1989) p. 293.

/4/ Wagner, R., Field-ion Microscopy Springer-Verlag, Berlin (1982).

15/ Garcia, N., ed., Scanning Tunneling Microscopy 86, North Holland, Amsterdam (1987).

16/ Bourret, A., and Bacmann, J.J., Surface Science, 162 (1985) 495.

/7/ Bourret, .A., Rouvière, J.L. and Penisson, J.M., Acta Cryst. A44 (1988) 838.

18/ Cullis, A.G., Chew, N.G., Irvine, S.J.C. and Giess, J., Inst. Phys. Conf. Ser. N 87 (IOP Publishing Ltd) (1987) 141

19/ Rouvière, J.L., Thesis Grenoble University (1989).

/10/ Karlsson, L. and Norden, H., Acta Metall. 36 (1988) 13 and 25.

/11/ Ichinose, H. and Ishida, Y., Phil. Mag. in press.

/12/ Ourmazd, H., and Taylor, D.W., Cunningham, J. and Tu, C.W., Phys. Rev. Lett. 62 (1989) 933.

113/ Howe, J.M., Laughlin, D.E. and Vasudevan, A.K., Phil. Mag. A57 (1988) 955.

/14/ Ichinose, H., Furuta, T., Sakaki, H. and Ishida, Y., Proc. of XIth Int. Congress on Electron Microscopy, Kyoto (The Japanese Society of Electron Microscopy, Tokyo), (1986) 1483.

15/ Nakamura, T., Ikeda, M., Muto, S, and Umebu, I., Appl. Phys. Lett. 53 (1988) 379.

/16/ Seidman, D.N., in Characterization of the Structure and Chemistry of Defects in Materials, ibid p. 315.

/17/ Bolin, P.L., Bayurick, R.J., Ranganathan, B.N., Phil. Mag. 31 (1975) 891.

118/ Balluffi, R.W., Majid, I. and Bristowe, P.D., in Characterization of the Structure and Chemistry of Defects in Materials, ibid p. 457.

119/ Waugh, A.R., Southon, M.J., Surface Sci. 68 (1977) 79.

120/ Bach, P.W., Beyer, J. and Verbreak, C.A., Scripta Met. 14 (1980) 205.

121/ Herstchitz, R. and Seidman, D.N., Acta Metal1 33 (1985) 1565.

/22/ Herstchitz, R. and Seidman, D.N., J. de Physique C5, 49 (1988) 469.

123/ Kazmerski, L.L., in Polycristalline semiconductors, ibid. p. 96.

/24/ Bel1, L.D., Kaiser, W.J., J. of Microscopy 152 (1988) 605. 
/25/ Fitzsimmons, M.R. and Sass, S.L., Acta Metal1. 36 (1988) 3103.

126/ Fitzsimmons, M.R. and Sass, S.L., Acta Metall 37 (1989) 1009.

/27/ Pate1, J.R., Golovchenko, J.A., Bean, J.C. and Morris, R.J., Phys. Rev. B 31 (1985) 6884 .

/28/ Bekzyk, M.J., Bilderback, D., White, J., Abrûna, H.D. and Bommarito, M.G., J. Phys. Chem. 90 (1986) 4926.

129/ Heald, S.M., Tranquada, J.M., J. Appl. Phys. 65 (1989) 290.

130/ Bourret, A. and Bacmann, J.J., Trans. Japan Inst. Met. Suppl. 27 (1986) 125.

/31/ Mauger, A., Bourgoin, J.C., Allan, G., Lannoo, M., Bourret, A. and Billard, L., Phys. Rev. B 35 (1987) 1267 .

/32/ Paxton, A.T. and Sutton, A.P., Acta Metal1 37 (1989) 1693.

/33/ Kohyama, M., Yamamoto, R., Watanabe, Y., Ebata, Y. and Kinoshita, M., J. Phys. C 21 (1988) L 695.

/34/ Bourret, A., Rouvière, J.L., in Polycristalline semiconductors, ibid p. 8.

135/ Rouvière, J.L. and Bourret, A., in Polycristalline semiconductors, ibid p. 19.

/36/ Krakow, W., Wetzel, J.T. and Smith, D.A., Phil. Mag. A 53 (1986) 739.

137/ Pénisson, J.M., Nowicki, T. and Biscondi, M., Phil. Mag. A 58 (1988) 947.

/38/ Nowicki, T., Thesis St Etienne University (1989).

/39/ Merkle, K.L. and Smith, D.J., Ultramicroscopy 22 (1987) 57.

$140 / \mathrm{Oh}, Y$. and Vitek V., Acta Metall. 34 (1986) 1941.

/41/ Hsieh, T.E. and Balluffi, R.W., Acta Metali. 37 (1989) 2133.

/42/ Leroux, Ch., Loiseau, A., Cadeville, M.C. and Ducastelle, F., submitted to Phys. Rev. Lett.

/43/ Fitzsimmons, M.R., Burke1, E. and Sass, S.L., Phys. Rev. Letters 61 (1988) 2237.

144/ Dahmen, U., Pelton, A.R., Whitcomb, M.J. and Westmacott, K.H. in Mater. Res. Soc. Proc. 52 (1986) 217 .

145/ Castano, V.M. and Krakow, W., Surface Science, 191 (1987) 45.

/46/ Penisson, J.M. and Regheere, G., J. of Mat. Science and Engineering, A 107 (1989) 199.

/47/ Zhang, S.Y. and Cowley, J.M., Thin Solid films 148 (1987) 301.

/48/ Kuwabara, M., Spence, J.C.H. and Ruhle, M., J. Mater. Res. 4 (1989) 972.

/49/ Mellul, S., Thesis Paris VI University 1989.

/50/ Hutchinson, J.L., in Inst. Phys. Conf. Ser. $N^{\circ} 87$ (IOP Publishing Ltd) (1987) 1.

/51/ Kim, Y., Ourmazd, A., Bode, M. and Feldman, R.D., Phys. Rev. Lett. (in press).

/52/ Bourret, A. and Rouvière, J.L., submitted to Phil. Mag.

/53/ Fujita, H., Taguchi, E. and Lu, C., J. Electron Micros. 37 (1988) 329.

/54/ Broniatowski, A., Phys. Rev. Lett. (in press).

/55/ Pinet-Berger, J.J. and Laval, J.Y., in "Ceramic microstructures" Edited by Pask and Evans (Plenum Publishing Company), (1988) 657

/56/ Elkajbaji, M. and Thibault-Desseaux, J., Phil. Mag. A 58 (1988) 325 .

/57/ Sutton, A.P., Phil. Mag. A60 (1989) 147.

/58/ Queisser, H.J. and Werner, J.H., Mat. Res. Soc. Symp. Proc. 106 (1988) 53. 